\title{
Geometrically Mediated Breakup of Drops in Microfluidic Devices
}

\author{
D. R. Link, ${ }^{1,2}$ S. L. Anna, ${ }^{2}$ D. A. Weitz, ${ }^{1,2}$ and H. A. Stone ${ }^{2}$ \\ ${ }^{1}$ Department of Physics, Harvard University, Cambridge, Massachusetts 02138, USA \\ ${ }^{2}$ DEAS, Harvard University, Cambridge, Massachusetts 02138, USA
}

(Received 29 May 2003; published 6 February 2004)

\begin{abstract}
Microfluidic technology offers capabilities for the precise handling of small fluid volumes dispersed as droplets. To fully exploit this potential requires simultaneous generation of multiple size droplets. We demonstrate two methods for passively breaking larger drops into precisely controlled daughter drops using pressure-driven flow in simple microfluidic configurations: (i) a $\mathrm{T}$ junction and (ii) flow past isolated obstacles. We quantify conditions for breakup at a $\mathrm{T}$ junction and illustrate sequential breakup at $\mathrm{T}$ junctions for making small drops at high dispersed phase volume fractions.
\end{abstract}

An emulsion consists of two immiscible fluids, one of which is dispersed as droplets in the continuous phase of the other. Typically, a surfactant is essential to stabilize the droplets against coalescence as they are thermodynamically metastable. Conventional emulsification techniques use inhomogeneous extensional and shear flows to rupture droplets; as a result, they generate emulsions with wide distributions in droplet sizes. However, many important potential uses of emulsions require precise control over droplet size and polydispersity, the standard deviation in droplet radius divided by the average radius. For example, emulsions with low polydispersity have been used to produce new materials where the droplets spontaneously order into regular packings that act as templates for sol-gel chemistry [1] or encapsulate electro-optical materials such as liquid crystal [2]. To overcome this limitation, several techniques have been developed to reduce polydispersity, including repeated fractionation of a polydisperse emulsion [3], and direct generation of uniform emulsion droplets by shearing immiscible fluids between uniformly separated plates [4].

Highly uniform emulsion droplets can alternatively be generated by single-drop technologies such as flow through a micromachined comb [5], hydrodynamic flow focusing through a small orifice [6], and drop break off in coflowing streams [7]. Although the production rate is limited, these techniques can benefit from the potential of highly parallel production offered by microfluidic technology, which reduces this limitation $[8,9]$.

However, full exploitation of microfluidic production and control of emulsion droplets is severely limited if only a single droplet size can be produced by each orifice; instead, much greater flexibility in potential usefulness would be afforded by methods to further adjust droplet size. This would facilitate the use of droplets as micromixers [10] and chemical reactors [11]; one advantage of a microfluidic design is that time after droplet formation corresponds to distance down a channel, allowing for long signal integration times in monitoring reaction kinetics without compromising temporal resolution [11].
However, no microfluidic technology currently exists to precisely break droplets to control the size distribution.

In this Letter, we report a methodology for passively breaking droplets into multiple sizes with the relative number and size of each precisely fixed. Of the two techniques investigated, breakup is accomplished with maximum precision at a $\mathrm{T}$ junction [11] where daughter droplets of a predetermined size ratio are generated. This type of passive breakup can be repeated in succession without increasing the polydispersity of the droplets until drop radii of the order of the channel width are achieved. Even smaller droplets can be produced albeit at increasingly high flow rates. We present an analytical model to understand this breaking to nonbreaking transition in terms of stretching of drops in an extensional flow. For situations where use of minimal device real estate is important, a second passive breakup technique consisting of an obstacle placed in the channel has been developed to break a predetermined fraction of the droplets.

In our experiments, we disperse droplets of water in a continuous phase of hexadecane. A surfactant, sorbitan mono-oleate (Span 80), at $3 \mathrm{wt}$ \% is added to the hexadecane to stabilize the droplets against coalescence. This reduces the interfacial tension between distilled water and the surfactant-hexadecane solution to $\gamma=5 \mathrm{mN} / \mathrm{m}$, as measured using a ring tensiometer. Syringe pumps are used to control the infusion of the fluids into microfluidic devices fabricated in poly(dimethylsiloxane) (PDMS), a transparent elastomer, using soft lithography techniques [12]. In each device, droplets are initially generated upstream at a $\mathrm{T}$ junction [8], where the channels have a $30 \mu \mathrm{m}$ square cross section and the continuous phase, hexadecane (viscosity $\eta=8 \times 10^{-3} \mathrm{Pas}$ ), shears off droplets of distilled water one at a time to generate an inverse emulsion with a narrow distribution in droplet size. Although hexadecane swells PDMS, we do not observe changes in our devices or results during the several hours of a typical experiment. Smaller droplets are generated with higher flow rates of the oil; this precludes the direct generation of emulsions with a high volume 
fraction of droplets. Under typical operating conditions, we achieve polydispersities of less than 5\% and usually between $1 \%$ and $3 \%$, where we determine the standard deviation of radius divided by mean radius by image analysis of droplet sizes. Water drops do not adhere to the walls as they do in other wetting scenarios, allowing well-defined droplets to flow down the channels [13].

To tailor the droplet size and distribution, we exploit the extensional flow in the neighborhood of the stagnation point of a T-shaped junction to precisely break drops into smaller droplets, as shown schematically in Fig. 1(a). For any ratio of the initial drop length to the channel width, there is a critical flow rate above which the drops always break into two daughter droplets of equal size, as shown in Fig. 1(b). Sufficiently long droplets always break at the $\mathrm{T}$ junction independent of the flow rate. By contrast, smaller droplets do not break if they are traveling too slowly down the channel, and instead flow alternately into each of the side channels. This technique has the advantage that it is passive, requiring no moving parts. Although polydispersity might be expected to increase on droplet breakup, we do not detect any change, which confirms the precise control achieved by this technique.

A significant advantage of T-junction passive breakup is that it can be modified to facilitate asymmetric breakup of droplets; this is accomplished by adjusting the relative lengths, $\ell_{1}$ and $\ell_{2}$, of the side channels. To account for this behavior, we make the microfluidic analog of an electriccurrent-splitting device where the flow rate in each channel, $q_{1}$ and $q_{2}$, is analogous to an electric current, and the pressure drop $\Delta p$ is analogous to a voltage. To find the analog of resistance, we consider a fixed flow rate $q$ down a channel of width $w_{0}$, height $h$, and length $l$. The pressure drop is $\Delta p=\beta q \ell$, where $\beta \approx \eta(3 / 4)\left[1 /\left(w_{0} h^{3}\right)\right] \times$ $\left[1-6(2 / \pi)^{5}\left(h / w_{0}\right)\right]^{-1}$; the electric circuit analog to the resistance is then $\beta \ell$. As $\beta$ is small in the wide section of the channel, the length of the wide section can be neglected in comparison to the narrow section, and the ratio of the flow rates in the arms is then inversely proportional

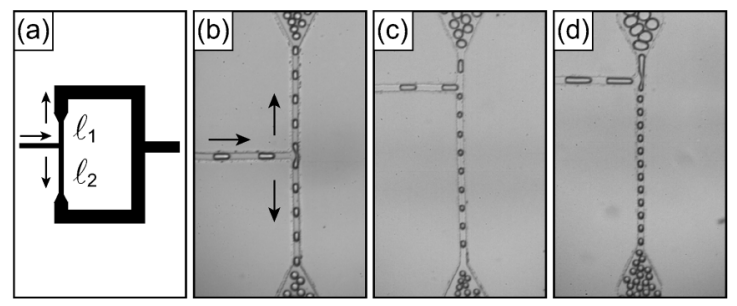

FIG. 1. Passive breakup at T junctions. Droplets flow into a T junction, sketched in (a), where the flow is split into two sidearms. The narrow portion of the two side arms, of lengths $\ell_{1}$ and $\ell_{2}$, control the relative flow resistance of the paths and, hence, the relative sizes of the two daughter drops as shown in the photomicrographs of (b), (c), and (d); the ratios of arm lengths are, respectively, $\ell_{1} / \ell_{2}=1: 1,1: 5.2$, and 1:8.1, corresponding to daughter drop volume ratios of (b) $1: 1$, (c) 1:5.2, and (d) 1:7.5. to the ratio of lengths $q_{1} / q_{2} \approx \ell_{2} / \ell_{1}$. In addition, the ratio of volumes of the two daughter droplets, $V_{1}$ and $V_{2}$, is proportional to the ratio of volume flow rates. Experimentally, we find that the ratio of volumes of the two daughter droplets is in good agreement with the expected ratio of sidearm lengths. From the images in Figs. 1(c) and 1(d), relative sidearm lengths $\ell_{2} / \ell_{1}$ of 5.2 and 8.1 result in daughter droplets with relative volumes $V_{1} / V_{2}$ of 5.2 and 7.5. The slight discrepancy in the second case may be due to a change in $\beta$ in the long channel due to the presence of a large number of droplets.

In order to fully exploit the potential of passive breakup, we must elucidate the physics of how the drops break, and determine the conditions under which they break. This can be done by examining both nonbreaking and breaking conditions. Before entering into the $\mathrm{T}$ junction, droplets have an initial extension $\varepsilon_{0}$ defined as the ratio of their length $\ell_{0}$ to circumference $\pi w_{0}$ and move at a velocity $v$ as indicated in Fig. 2(a). The case of nonbreaking drops moving close to the critical velocity for breaking is shown in Figs. 2(a)-2(d). In Fig. 2(a), a droplet of $\varepsilon_{0} \approx 0.4$ enters the $\mathrm{T}$ junction, begins to stretch in Fig. 2(b), reaches a maximum extension in Fig. 2(c), and moves out of the T junction in Fig. 2(d). An enlargement of the drop at its point of maximum extension $\varepsilon=$ $\ell_{e} /\left(\pi w_{e}\right)=0.95$ is shown in Fig. 2(e), where $\ell_{e}$ and $w_{e}$ are, respectively, the stretched drop's length and width. For conditions where drops break, the images in Figs. 2(f)-2(i) show a slightly longer drop moving at a slightly greater velocity as it enters into the $\mathrm{T}$ junction. In this case, the drop is stretched to a maximum length in Fig. 2(g) and then begins to break in Fig. 2(h) with the daughter drops moving in opposite directions in Fig. 2(i). The maximum extension is $\varepsilon=1.15$ as shown in Fig. 2(j). In fact, we observe that drops stretched beyond $\varepsilon=1$ always break, while drops with maximum extensions below unity never break, except in cases where the droplets are closely spaced and collide in the $\mathrm{T}$ junction. This limit is consistent with the classical Rayleigh-Plateau instability in which a cylindrical liquid thread can reduce its total surface area by breaking when its length exceeds its circumference. In the case of asymmetric breakup, the high pressure point near the center of the $\mathrm{T}$ junction sets the location where the droplet breaks.

Since the deformation rate required to stretch a small drop to and beyond $\varepsilon=1$ is greater than that required to stretch a large drop beyond this limit, the critical velocity necessary to break a drop depends on its size. We define the capillary number $C$ as the ratio of viscous stresses to those due to interfacial tension, $C=\eta v / \gamma$. Then, using various flow conditions, we determine $C$ as a function of the measured initial extension of the droplets $\varepsilon_{0}$, and plot the results in Fig. 2(k), distinguishing drops that break (open circles) from those that do not (solid squares). Consistent with our expectations, drops whose initial extension values are greater than one, $\varepsilon_{0}>1$, always break in the $\mathrm{T}$ junction, even at the lowest achievable capillary 


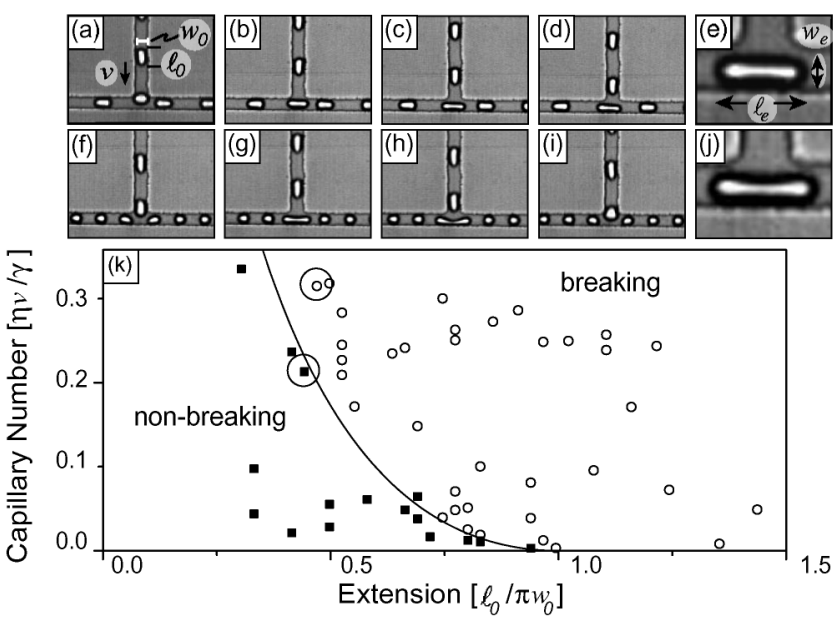

FIG. 2. Critical conditions for breaking drops at $\mathrm{T}$ junctions. In a channel of width $w_{0}$, droplets having length $\ell_{0}$ and velocity $v$ enter a $\mathrm{T}$ junction and either break or do not break. An example of nonbreaking drops, corresponding to the circled point below the curve in (k), enter the T junction in (a), stretch in (b), reach a maximum extension in (c), and then move alternatively left and right out of the junction in (d). At maximum extension, shown in (e), the extended length $\ell_{e}$ and width $w_{e}$ give $\varepsilon=\ell_{e} /\left(\pi w_{e}\right)=0.95$. A similar series for breaking conditions corresponding to the circled point above the critical line are shown in (f)-(i). In this case, the maximum extension shown in (j) is $\varepsilon=1.15$. In $(\mathrm{k})$, open circles indicate when drops of a given capillary number $C=\eta v / \gamma$ and initial extension $\varepsilon_{0}=\ell_{0} /\left(\pi w_{0}\right)$ break, and squares indicate when they do not. A model for the critical capillary number for breaking $C_{\mathrm{cr}}$ provides the curve $C_{\mathrm{cr}}=\alpha \varepsilon_{0}\left(1 / \varepsilon_{0}^{2 / 3}-1\right)^{2}$ shown in (k) with fitting parameter $\alpha=1$.

numbers. However, they never break upstream in the straight channel preceding the $\mathrm{T}$ junction, despite being longer than the Rayleigh-Plateau limit. We believe this stability is due to the flow around the drop, which convects disturbances toward the end of the drop before they can grow, and the nearby walls, which act to suppress growth of instabilities.

The critical capillary number separating regions of breaking and nonbreaking drops in Fig. 2(k) can be understood by considering how droplets are stretched in an extensional flow of stretch rate $G$. Here we define an extensional flow capillary number $C_{G}=\eta G a / \gamma$, where $a$ represents the undeformed radius of the drop. For an extensional flow in the neighborhood of a $\mathrm{T}$ junction formed from channels of width $w_{0}$, the stretch rate is $G \approx$ $v / w_{0}$, or $C_{G}=C a / w_{0}$. Extensional stresses at the stagnation point, which are much larger than the shear stresses upstream of the junction, change the drop length from $\ell_{0}$ to $\ell_{e}$ [14]. Since we are seeking the critical conditions at which the drop just breaks, we write $\ell_{e}-$ $\ell_{0} \propto\left(G-G_{0}\right)^{1 / 2}$, where $G_{0}$ is the upstream shear rate (Eq. 8 of Ref. [14]). Then with $G_{0} \ll G$, the capillary number $C_{G}$ for breakup is $C_{G} \propto\left(\ell_{e} / a-\ell_{0} / a\right)^{2}$. We take $\ell_{e} / \pi w_{e}=1$, since the drop is at the stability limit, and we impose volume conservation, $\ell_{0} w_{0}^{2}=\ell_{e} w_{e}^{2}$, to obtain

054503-3 an expression for the critical capillary number $C_{\mathrm{cr}}$ for breaking a drop in the T junction: $C_{\mathrm{cr}}=\alpha \varepsilon_{0}\left(1 / \varepsilon_{0}^{2 / 3}-1\right)^{2}$, where $\alpha$ is a dimensionless constant, which is a function of the viscosity contrast of the two fluids and the geometry of the channel. This functional form for $C_{\mathrm{cr}}$ versus $\varepsilon_{0}$ is shown in Fig. 2(k) with a fitted constant of proportionality of $\alpha=1$. The predicted criteria for breakup is in excellent agreement with the experimentally observed transition. Finally, for the case of asymmetric configurations, the Rayleigh-Plateau stability criterion may need to be adjusted to compensate for the fact that the disturbance to the extended drop shape is no longer at the center of the drop. In particular, we expect asymmetric drops, such as the breakup shown in Figs. 1(c) and 1(d), to be stable at larger extensions than symmetric drops since the smaller daughter drop will not break until the circumference of the original extended drop has become sufficiently small; this has been confirmed qualitatively.

Using this understanding, we implement a series of $\mathrm{T}$ junctions in which drops are successively broken to independently manipulate both droplet size and dispersed phase volume fraction in a given emulsion. Using dropby-drop emulsification techniques such as $\mathrm{T}$ junctions [8], it is not possible to directly make small droplets at high dispersed phase volume fractions. However, as shown in Fig. 3(a), sequential application of the geometrically mediated T-junction breakup facilitates the precise conversion of large initial slugs of the dispersed phase into small droplets comparable in size to the channel width without altering the dispersed phase volume fraction. The figure illustrates a series of three T-junction mediated breakup events, in which every long slug is reduced into eight small droplets, after which the eight exit arms are recombined to form a single densely packed emulsion as shown in Fig. 3(b). The deformation of densely packed droplets and a nonuniform channel depth due to swelling of PDMS by hexadecane gives the impression in Fig. 3 that the polydispersity is higher than the $3 \%$ that we measure when the drops are collected.

In some applications, the T-junction design may require too much space on the device or applied pressures

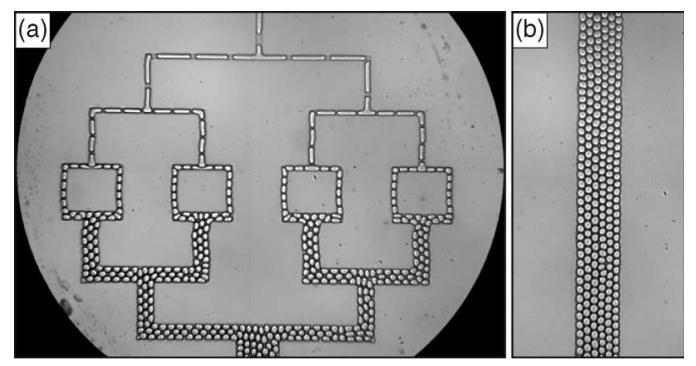

FIG. 3. Sequential application of passive breakup. In (a) drops are formed at high dispersed phase volume fractions (drop formation not shown) and are sequentially broken to form small drops. In (b) the droplets flow downstream with nearly defect-free hexagonal-close-packed ordering. 
that exceed device capabilities. An alternative passive breakup configuration, which overcomes these limitations, uses flow past an obstacle to effect controllable changes in drop size. Here, an obstruction is placed downstream of the region where drops are first formed, as illustrated in Fig. 4(a). The transverse placement of the obstacle, indicated by the distances $a$ and $b$ from each sidewall, allows control over the degree of breakup and the relative size distribution of the resulting dispersion. In the obstruction-mediated breakup devices considered here, the obstacle is a $60 \mu \mathrm{m}$ square box, the distance $a$ from the sidewall is fixed at $15 \mu \mathrm{m}$, and the distance $b$ from the opposite wall varies such that the ratio $a / b$ is $1: 1.2$ or $1: 2.7$. Thus, the total channel widths in the neighborhood of the obstacle vary from 90 to $120 \mu \mathrm{m}$.

We observe three aspects of drop breakup in pressuredriven flow past an obstacle. In the first, a single column of drops, with size comparable to the channel, flows past an obstacle centered in the channel. The drops deform as they flow in the narrow gaps and break into two daughter drops, as shown in Fig. 4(b). Changing the asymmetric location of the obstacle allows control of the relative sizes of the daughter droplets, as in Fig. 4(c). In addition, changes of the packing configuration occur downstream of the obstacle. The choice of the geometric shape of the channel upstream of the obstacle, e.g., the presence of an expansion downstream of the $\mathrm{T}$ junction where drops are first formed, and the relative flow rates of the two liquid phases also allow for the possibility of drops flowing single file or in multiple columns as the obstruction is approached. When a two-layer configuration of droplets encounters an obstacle placed off center, only drops in one of the layers breaks, resulting in a regular sequence of three different sizes of drops, illustrated in Fig. 4(d). Similar results can also be achieved with other patterns, such as three rows where every third droplet breaks.

The two breakup techniques described above each have advantages and disadvantages. Since it is easier to fabricate channels of precise length than a narrow gap, the $\mathrm{T}$ junction is more reliable than the single obstruction design. In addition, the $\mathrm{T}$ junction offers the possibility of actively controlling drop size distribution. Rather than adjusting the relative arm lengths to set the desired ratio of daughter drop volumes, the relative flow resistance can instead be controlled by changing the cross-sectional dimensions using pressure-actuated valves [15] or by adjusting the exit pressures in the two arms [11]. For the obstruction-mediated breakup design, the relative sizes of the resulting daughter drops are sensitive to the precise lateral placement of the obstacle, as well as the location of the incoming drops, making it more difficult to control. Another potential drawback of this design is that the initial droplet pattern must span the width of the channel so that drops are forced to deform around the obstacle rather than simply pass through the narrow gaps. Despite these drawbacks, this design has significant advantages. Since only a single obstacle is placed in the flow, the total

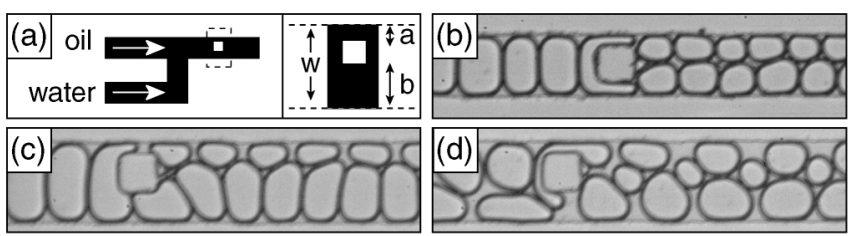

FIG. 4. Obstruction mediated passive breakup. (a) Schematic diagram of a square obstruction in a microchannel. The obstacle is a $60 \mu \mathrm{m}$ square; $a=15 \mu \mathrm{m}$. (b) The obstacle is approximately in the center of the channel so that the ratio $a / b$ is $1: 1.2$. (c) The channel width is $120 \mu \mathrm{m}$ and the ratio $a / b$ is 1:2.7. (d) As in (c) but every second drop breaks when a twolayer pattern encounters an off-center obstruction.

pressure drop along the channel is not substantially affected. Also, small obstructions require significantly less real estate than $\mathrm{T}$ junctions, making obstructions attractive for applications where precision can be sacrificed for efficiency.

Using the concept of geometrically mediated drop breakup, we have demonstrated the ability to design dispersions with controlled volume fractions and drop sizes. Each passive path to drop size reduction that we present here is fabricated using soft lithography methods and allow precise manipulation of drop volume in conjunction with any initial drop size distribution.

This work was supported by Unilever and the Harvard MRSEC (DMR-0213805). We thank T. Jongen and colleagues for helpful discussion, the G. M. Whitesides group for assistance in learning soft lithography, and G. H. McKinley for use of their tensiometer.

[1] A. Imhof and D. J. Pine, Nature (London) 389, 948 (1997).

[2] D. Rudhardt, A. Fernández-Nieves, D. R. Link, and D. A. Weitz, Appl. Phys. Lett. 82, 2610 (2003).

[3] J. Bibette, J. Colloid Interface Sci. 147, 474 (1991).

[4] T. G. Mason and J. Bibette, Phys. Rev. Lett. 77, 3481 (1996).

[5] S. Sugiura, M. Nakajima, S. Iwamoto, and M. Seki, Langmuir 17, 5562 (2001).

[6] A. M. Gañán-Calvo, Phys. Rev. Lett. 80, 285 (1998)

[7] P. B. Umbanhowar, V. Prasad, and D. A. Weitz, Langmuir 16, 347 (2000).

[8] T. Thorsen, R.W. Roberts, F. H. Arnold, and S. R. Quake, Phys. Rev. Lett. 86, 4163 (2001).

[9] S. L. Anna, N. Bontoux, and H. A. Stone, Appl. Phys. Lett. 82, 364 (2003).

[10] M. Burns et al., Science 282, 484 (1998).

[11] H. Song, J. D. Tice, and R. Ismagilov, Angew. Chem., Int. Ed. Engl. 42, 768 (2003).

[12] J.C. McDonald et al., Electrophoresis 21, 27 (2000).

[13] R. Dreyfus, P. Tabeling, and H. Willaime, Phys. Rev. Lett. 90, 144505 (1993).

[14] Y. Navot, Phys. Fluids 11, 990 (1999).

[15] M. A. Unger et al., Science 288, 113 (2000). 